\title{
BMJ Open Protocol for a systematic review and meta-analysis of fractional dose compared with standard dose inactivated polio vaccination in children
}

\author{
Thandiwe Runyararo Mashunye, ${ }^{1}$ Duduzile Edith Ndwandwe, ${ }^{2}$ \\ Kopano Rebaona Dube, ${ }^{2}$ Muki Shey, ${ }^{3}$ Mary Shelton, ${ }^{4}$ Charles Shey Wiysonge ${ }^{\oplus, 2,5}$
}

To cite: Mashunye TR, Ndwandwe DE, Dube KR, et al. Protocol for a systematic review and metaanalysis of fractional dose compared with standard dose inactivated polio vaccination in children. BMJ Open 2019;9:e023308. doi:10.1136/ bmjopen-2018-023308

- Prepublication history for this paper is available online. To view these files, please visit the journal online (http://dx.doi org/10.1136/bmjopen-2018023308).

Received 30 March 2018 Revised 29 January 2019 Accepted 31 January 2019

Deck for updates

(C) Author(s) (or their employer(s)) 2019. Re-use permitted under CC BY-NC. No commercial re-use. See rights and permissions. Published by BMJ.

For numbered affiliations see end of article.

Correspondence to Dr Thandiwe

Runyararo Mashunye;

tmashunye1@gmail.com

\section{ABSTRACT}

Introduction WHO recommends the introduction of at least one single dose of inactivated polio vaccine (IPV) in routine immunisation schedules. Thus, there has been an increased demand and concurrent supply shortages of IPV worldwide. One of the strategies to improve access is the use of fractional instead of full doses of IPV. We aim to compare the effects of fractional with standard doses of IPV.

Methods and analysis We will include randomised trials, non-randomised trials, case-control studies and cohort studies that compared fractional with full doses of IPV among children aged 5 years or younger. We will search for eligible studies among published and grey literature. Two authors will independently screen the results of the search, select studies, extract data and assess risk of bias. We will stratify analyses by study design, type of poliovirus, type of outcome measure and number of IPV doses given. For each type of poliovirus, we will pool the outcome data from studies using random-effects meta-analyses. Statistical heterogeneity will be assessed using the $\chi^{2}$ test of homogeneity and quantified using the $I^{2}$ statistic. To investigate statistical heterogeneity, subgroup analyses will be performed based on the timing of the first fractional dose, age of administration, immunisation schedules and country income status. Sensitivity analyses will be used to assess if the effect of IPV fractional dosing is affected by study design, risk of bias and methods of meta-analysis. Ethics and dissemination We obtained approval from the University of Cape Town Human Research Ethics Committee (HREC REF: 412/2018). The findings of this review will provide evidence for decision-making with regards to IPV dosage, eventually improving access to the vaccine by stretching vaccine supplies. The results will be published in the University of Cape Town online library and in a peer reviewed journal.

PROSPERO registration number CRD42018092647.

\section{INTRODUCTION}

Poliomyelitis (or polio) is a communicable disease caused by one of three related wild polioviruses: poliovirus types 1,2 and 3 . Infection can occur at any age but it mainly affects children under 5 years of age. ${ }^{1}$ The
Strengths and limitations of this study

- We will conduct study selection, data extraction and risk of bias assessment in duplicate to minimise the risk of bias.

- We will use the Grading of Recommendations Assessment, Development and Evaluation approach to evaluate the certainty of the evidence for each outcome.

- Since non-randomised studies will be included, we anticipate high risk of selection bias, which will be mitigated by conducting sensitivity analyses.

virus typically enters the body through the mouth and multiplies inside the gut. Initially, it manifests as flu-like symptoms. Once established, it enters the bloodstream and attacks the central nervous system. As it proliferates, it destroys nerve cells which stimulate muscles. These nerve cells cannot be renewed and affected muscles no longer function, causing paralysis. Up to $95 \%$ of infected individuals have no symptoms and about $5 \%$ who develop minor flu-like symptoms fully recover. ${ }^{2}$ Paralysis occurs in less than $1 \%$ of infected individuals. ${ }^{2}$ Immunity against polio is acquired following recovery from a natural infection with poliovirus or vaccination with a polio vaccine. There are two types of polio vaccines, which are highly effective. The first is an injectable vaccine developed in the 1950s (the inactivated polio vaccine [IPV]) and the second, an oral vaccine developed in the 1960s (the oral polio vaccine [OPV]). ${ }^{1}$

In the early 1980s, more than 350000 cases of paralytic polio were estimated to occur per year worldwide. ${ }^{3}$ The widespread use of the OPV resulted in substantial advances towards eradicating polio. ${ }^{3-5}$ However, the vaccine has been linked to vaccine-associated paralytic poliomyelitis (VAPP) and the generation of vaccine-derived polioviruses (VDPVs). ${ }^{6}{ }^{7}$ 
These liabilities threaten the achievement of a polio-free world. The Polio Eradication and Endgame Strategic Plan 2013-2018 outlines the necessary steps required to ensure that transmission of both wild polio viruses (WPV) and VDPVs is interrupted. One of its objectives is to prevent the spread and re-emergence of VDPVs by gradually replacing OPV with the IPV. ${ }^{8}$ The first stage in the phased removal of OPV was completed in April 2016. ${ }^{9}$ It involved the cessation of the type 2 component of OPV through a global switch from trivalent OPV (containing all three poliovirus types) to bivalent OPV (containing only poliovirus types 1 and 3). ${ }^{8}$

Prior to switching, in November 2012, the Strategic Advisory Group of Experts on Immunisation recommended the introduction of at least one dose of IPV in national immunisation schedules to mitigate the risk of re-introduction or re-emergence of WPV type 2 or VDPV type $2 .{ }^{10}$ This minimum dose was meant to provide an immune base to improve immune response and lower the risk of paralysis in the event of a type 2 polio outbreak. ${ }^{311}$ In its 2016 polio vaccine position article, the WHO endorsed the recommendation. ${ }^{3}$ Globally, the demand for IPV increased from 80 million doses in 2013 to 200 million doses in 2016. ${ }^{12}$ The global manufacturers only managed to supply about half of the required doses for 2016 and 2017 and the supply is deteriorating. ${ }^{12}{ }^{13}$ With the limited supply and lack of competition in the market, the cost of IPV (up to US\$2.8 per dose) ${ }^{13} 14$ further restricts access in resource-constrained countries.

IPV shortages and high cost have given rise to delayed IPV introduction in some countries, while countries that already have IPV as part of their schedule are experiencing stock outs. ${ }^{15}{ }^{16}$ By September 2016, about 105 out of 126 countries that had OPV-only immunisation schedules had introduced IPV.$^{17}$ According to an update from Unicef in March 2017, there were 18 countries that had introduced IPV which were only to receive IPV shipments in the first half of $2018 .{ }^{18}$ Going forward, after the expected certification of global polio eradication in 2022, IPV will be the only vaccine being used. ${ }^{19}$ This puts pressure on both the global community and individual countries to come up with strategies to ensure affordable and reliable longterm supply of IPV.

To address the current shortages and ensure availability of IPV, the Global Polio Eradication Initiative and its partners are using a multipronged approach. ${ }^{16}$ One of the recommended strategies is the use of dose sparing fractional doses instead of full doses of IPV. ${ }^{36}$ One fractional dose $(0.1 \mathrm{~mL})$ is one-fifth of the full dose $(0.5 \mathrm{~mL})$ and it is usually delivered using an intradermal injection. Several randomised studies have assessed the immunogenicity of fractional doses of IPV (fIPV) compared with full dose(s) of IPV. ${ }^{20-23}$

Polio vaccination invokes a humoral immune response producing antibodies which, in most cases, offer longterm protection against polio viruses. ${ }^{3}$ Intradermal delivery of the polio vaccine is an efficient and effective mode of vaccination because it allows for the dose sparing approach. ${ }^{24}{ }^{25}$ Due to the abundance of immune cells in the dermis, when a vaccine is given via the dermis, a lower dose can be used compared with the intramuscular and subcutaneous routes. ${ }^{25}$ In terms of cost, the price of one-fifth of the dose is expected to be a fifth of the price of the full dose; hence, reducing the cost. ${ }^{4}$ According to an assessment that was done by PATH, two fIPV doses and the intradermal injection devices cost between US $\$ 1$ and US $\$ 3$ while a single intramuscular dose of IPV costs between US $\$ 1.1$ and US $\$ 2.3 .^{25}$

This dose sparing strategy might be the immediate solution to ensure that every child who is entitled to receive IPV is vaccinated. India and Sri Lanka (among other countries) have introduced the fIPV doses in their routine immunisation schedules with notable successes. Both countries have managed to vaccinate a larger number of eligible children while stretching their IPV vaccine stocks; subsequently, avoiding stock outs. ${ }^{16}$ A mass vaccination campaign using a single fIPV was successfully carried out in response to a VDPV type 2 outbreak in the Telangana State of India in May 2016. ${ }^{26}$

Countries are encouraged to consider the programmatic and logistic challenges that come with introducing fIPV before rolling out the policy. ${ }^{3}$ Intradermal administration involves purchasing of devices to administer, training health workers and other logistics which affect the feasibility of the programme. ${ }^{17}$ Programmes in India and Sri Lanka have demonstrated that despite these challenges, a nationwide programme using fIPV is possible even in an outbreak setting. ${ }^{1626}$

The Sustainable Development Goal number 3 includes achieving access to affordable, safe and effective vaccines for all by $2030 .^{27}$ Replacing OPV with IPV is essential for the eradication of WPV and VAPP. As we progress towards the certification of the global eradication of polio, ensuring sustainable, reliable and affordable IPV supply is of paramount importance. The dose sparing and cost reduction characteristics of fractionated doses can contribute to improved IPV access. This review will add to the evidence gathered by other reviews to inform decisions regarding the value of fractionated IPV dosages.

Grassly ${ }^{28}$ performed a systematic review and meta-analysis to assess the immunogenicity and effectiveness of 1 or 2 doses of fIPV in routine immunisation. The author found that two fIPV doses administered after 10 weeks of age are likely to provide $80 \%$ protection against poliomyelitis. It is now 6-7 years since the literature search was conducted for this review, and new evidence has accumulated since then. ${ }^{23}{ }^{26}$ In addition, study selection and data extraction were not done in duplicate; increasing the risk of systematic errors in this timely review. ${ }^{28}$ It is an indispensable practice in systematic reviews to conduct those critical steps in duplicate, and resolve discrepancies by discussion and consensus or arbitration. ${ }^{29}$

In another review, Anand et $a l^{11}$ focused on comparing the immunogenicity of two fIPV and one full dose of IPV. They concluded that two fIPV doses are more immunogenic than a single dose. Of note, in both reviews, ${ }^{11} 28$ 
there is absence of an extensive search of the literature. Grassly searched only the Web of Knowledge collection of databases and Anand and colleagues' search was restricted to the PubMed database. Therefore, there might be other studies that were missed by these reviews that may alter their findings. Vaccine safety and administration are important considerations when deciding on an immunisation schedule. The previous reviews did not evaluate adverse events following administration of different IPV dosages and the devices used to give the intradermal injections. In addition, none of the previous reviews used the Grading of Recommendations Assessment, Development and Evaluation (GRADE) method ${ }^{30}$ to assess the certainty of the evidence on effects of fIPV. The GRADE approach is now widely adopted as a standard for evidence assessment. ${ }^{31}$

We aim to do a systematic review of studies comparing the effects of fractional compared with full dose IPV vaccination. We will evaluate the occurrence of adverse events following vaccination and document the types of administration devices used for the intradermal delivery of fIPV.

\section{OBJECTIVES}

The objective of this review is to assess the effects of fIPV, compared with full doses of the vaccine.

\section{METHODS AND ANALYSIS}

Criteria for considering studies for this review

Types of studies

We will include randomised trials, non-randomised trials, case-control studies and cohort studies.

\section{Types of participants}

The participants of interest will be children aged 5 years of age or younger.

\section{Types of intervention}

The eligible intervention will be the administration of fIPV, while the eligible comparison will be the administration of full dose(s) of the vaccine; irrespective of vaccination schedule or route of administration. Only studies comparing the same number of doses of fractional versus full doses of IPV will be compared.

\section{Types of outcome measures}

The primary outcome for this review is immunogenicity, measured using the proportion of participants who sero-converted (as defined by the authors of the included studies) as well as titres of poliovirus-neutralising antibodies for wild poliovirus serotypes 1, 2 and 3; assessed at least 4 weeks following vaccination.

Our secondary outcomes include adverse events following polio vaccination, vaccine-associated paralytic polio, wild poliovirus-associated paralytic polio and mucosal immunity (as defined by the authors). We will also describe the routes and devices used to administer the fractional doses of the vaccine.
Box 1 Proposed search strategy for the PubMed database

\#1: Poliovirus Vaccine, Inactivated [MeSH] OR inactivated polio vaccine OR inactivated poliovirus vaccine OR SALK OR IPV OR eIPV OR killed vaccine.

\#2: Injections, Intradermal [MeSH] OR Injections, Intramuscular [MeSH] OR fractional dosing OR Fractionated dosing OR drug dose comparison OR intradermal OR intramuscular OR dose OR dosage.

\#3: Dose-Response Relationship, Immunologic [MeSH] OR Antibody Formation [MeSH] OR Seroconversion [MeSH] OR Immunogenicity OR Immune response $\mathrm{OR}$ Seroconversion $\mathrm{OR}$ potency $\mathrm{OR}$ antibody formation $\mathrm{OR}$ antibody response.

\#4: (\#1 AND \#2 AND \#3).

\#5: Animals NOT Human.

\#6: (\#4 NOT \#5).

elPV, enhanced inactivated polio vaccine; MeSH, Medical Subject Heading.

\section{Search methods for identification of studies}

We will develop a comprehensive search strategy for peer-reviewed and grey literature. We will search the following databases from their inception to the date of the search; with no date or publication restrictions: Cochrane Central Register of Controlled Trials (CENTRAL), PubMed/MEDLINE, EMBASE, Science Citation Index, Conference Proceedings Citation Index, Cochrane Database of Systematic Reviews, Database of Abstracts of Reviews of Effectiveness, Scopus, EBSCO Host: Africa-Wide Information, CINAHL, Health Source: Nursing/Academic Edition, PDQ-Evidence, Network of Digital Library of and Theses and Dissertation, DART Europe E-theses Portal, ProQuest Dissertations \& Theses A\&I, PapersFirst (OCLC), Proceedings (OCLC) and the WHO International Clinical Trials Registry Platform. In addition, we will search the reference lists of included studies, related reviews and relevant WHO vaccine position papers. Box 1 shows the proposed search strategy for the PubMed database, which will be adapted for the other databases. Two authors (Thandiwe Mashunye and Mary Shelton) developed the search strategy.

\section{Selection of studies}

Two authors (Thandiwe Mashunye and Kopano Dube) will independently screen the search output for potentially eligible studies. We will obtain full texts for all the potentially eligible studies. The two authors will assess these full-text publications for eligibility. We plan to translate full texts of potentially eligible studies which are not written in English before assessing for eligibility. Any disagreements between the two authors regarding study eligibility will be resolved by discussion and consensus. A third author (Duduzile Ndwandwe or Charles Wiysonge) will arbitrate any unresolved disagreements.

\section{Data extraction and management}

For each included study, two authors (Thandiwe Mashunye and Kopano Dube) will independently extract the information indicated in table 1 using a pre-designed and piloted data extraction form. Extracted data will include 
Table 1 Information that will be extracted from each included study

Study design and methods Citation information (authors, journal, year of publication, volume, issue and page numbers); study design (randomised trial, non-randomised trial, case-control study, cohort study); study location (city, country) and period of study (start and end date, ie month and year).

Methods for generating randomisation sequence, concealing allocation of interventions and blinding of outcome assessment; number of participants randomised and number with complete outcome data for each outcome; names of outcomes announced in study protocol but not reported in study publication, and other biases.

Participant characteristics Age, sex, study location, study inclusion and exclusion criteria, flow of numbers of participants during the study (from participant enrolment to completion of data collection).

Intervention

Definition of fractional dose, manufacturer of vaccine, immunisation schedule (frequency, timing, interval between doses, etc), OPV co-administration, route of administration (intradermal, subcutaneous, etc) and types of devices used for administration of the fractional doses (name, manufacturing company, etc).

\begin{tabular}{ll} 
Comparison & $\begin{array}{l}\text { Definition of full dose, manufacturer of vaccine, immunisation schedule (frequency, timing, interval } \\
\text { between doses, etc), OPV co-administration and route of administration (intramuscular, sub- } \\
\text { cutaneous, etc). }\end{array}$ \\
Outcome measures & $\begin{array}{l}\text { Outcomes reported in the study (immunogenicity, adverse events following polio vaccination, } \\
\text { vaccine-associated paralytic polio, wild poliovirus-associated paralytic polio, mucosal immunity), } \\
\text { how they were defined and how they were measured. }\end{array}$ \\
Outcome data & $\begin{array}{l}\text { Seroconversion (number of participants randomised and number who seroconverted in each arm), } \\
\text { antibody titres (geometric mean plus SD or median and range in each arm), number of participants } \\
\text { randomised and number who experienced the following events in each arm: adverse events } \\
\text { following IPV vaccination, vaccine associated paralytic polio, wild poliovirus-associated paralytic } \\
\text { polio, mucosal immunity. }\end{array}$ \\
\hline
\end{tabular}

study, participant, intervention and outcome characteristics as well as study findings (table 1). We will present this information in a table of the characteristics of included studies. Any differences will be resolved through discussion and consensus between the two authors. A third author (Duduzile Ndwandwe or Charles Wiysonge) will be consulted to arbitrate if disagreements persist between the two authors. If there are missing data, we will contact study investigators for the missing information.

\section{Assessment of risk of bias in included studies}

Two authors will independently assess the risk of bias for each included study using the Cochrane Risk of Bias tool for trials ${ }^{32}$ and the ROBINS-I tool for non-randomised studies of interventions. ${ }^{33}$ For trials, we will assess the risk of bias across seven domains: random sequence generation, allocation concealment, blinding of participants and study personnel, blinding of outcome assessment, completeness of outcome data, selective outcome reporting and other potential source of bias. For each included study, whether trial or not, we will describe what the study authors reported that they did for each domain and then assign a judgement of low, high or unclear risk of bias. Differences in judgement will be resolved by discussion and consensus, or arbitration by a third author. Based on these assessments, we will classify each included study as having a low, moderate or high risk of bias. Each study that receives a judgement of high risk for allocation concealment, blinding of outcome assessment or completeness of outcome data will be considered to have a high risk of bias. A study that is judged to have low risk of bias for all three domains will be considered to have a low risk of bias. All other studies will be considered to have a moderate risk of bias. We will use these risks of bias data in the GRADE assessments of certainty of evidence, as described below, by downgrading the certainty of the evidence of effects from trials with high risk of bias.

\section{Dealing with missing data}

For incomplete or missing data, we will contact corresponding authors to request additional information or clarification. Missing data and dropouts will be reported in the 'risk of bias' table and we will evaluate the impact of missing data on our results. Where possible we will conduct intention to treat analysis. We will conduct sensitivity analyses whereby missing data are treated as successes or failures (worst-best and best-worst case scenario sensitivity analyses).

\section{Data synthesis}

We will stratify the analyses by the type of study design, type of poliovirus, type of outcome measure and number of IPV doses given. We will use the risk ratio and its corresponding $95 \%$ CI to summarise data for the following binary outcome measures: seroconversion, adverse events following polio vaccination, vaccine-associated paralytic polio and wild poliovirus-associated paralytic polio. For geometric median titres (our only outcome measure with continuous data), we will calculate the mean difference (MD) and its 95\% CI when the outcome data are measured on the same scale. Alternatively, if the measurement scale differs, the standardised mean difference 
(SMD) and its 95\% CI will be used. The alpha level of significance will be 0.05 .

For each type of poliovirus, we will pool the outcome data from studies with the same design and number of fractional versus full IPV doses administered using the random-effects method of meta-analysis. In studies where there are multiple interventions, we will include pair-wise comparisons that address the objectives of our review as outlined above. To avoid double counts when analysing correlated groups, we will pool the groups together to create a single pair-wise comparison.

\section{Assessment of heterogeneity}

Initially, we will assess for heterogeneity by visually inspecting the forest plots for overlapping CI. To further evaluate statistical heterogeneity, we will use the $\chi^{2}$ test of homogeneity; with significance defined at the alpha level of 0.10 . We will also use the $\mathrm{I}^{2}$ statistic to quantify the amount of heterogeneity. ${ }^{34}$ We will investigate the causes of statistical heterogeneity using subgroup analyses.

We will define subgroups based on the timing of the first fractional dose, age of administration, number of fractional doses, interval between doses, poliovirus type (1, 2 and 3), type of intradermal injection device, immunisation schedules (OPV containing versus non-OPV schedules) and country income status. ${ }^{35}$

\section{Assessment of reporting biases}

We will reduce possible publication bias by using a comprehensive search strategy. Literature sources will include published, unpublished and grey literature. To investigate possible publication bias, we will construct funnel plots if there are more than 10 studies included in the meta-analysis. ${ }^{36}$

\section{Sensitivity analysis}

We will perform sensitivity analyses to assess if the effect of fIPV will be affected by risk of bias (by excluding studies with a high risk of bias), study designs (randomised vs non-randomised) and methods of meta-analysis (fixed vs random effects).

\section{Reporting review findings}

We have written this protocol, and we will report the outcome, following the recommendations of relevant Preferred Reporting Items for Systematic Reviews and Meta-Analyses guidelines. ${ }^{37-39}$ In addition, forest plots and GRADE summary of findings tables will be used to report the outcome of our analysis. We will use the GRADE approach to evaluate the certainty of the evidence.

The GRADE approach judges the certainty of a body of evidence as high, moderate, low or very low; through appraising the risk of bias, imprecision, inconsistency and indirectness of study results, and the risk of publication bias. ${ }^{40}$ Regarding risk of bias, concerns that will affect our confidence in the evidence of effects are lack of allocation concealment, lack of blinding of outcome assessors and differential losses to follow-up of more than $10 \%$ between fractional and full dose groups. Inconsistency of effects across included studies, for which we find no compelling explanations, will reduce our confidence in the evidence. We will consider indirectness to exist if there are differences between the participants, interventions, comparisons and outcomes of our review, and those reported in included studies. We do not anticipate that this will be an issue in our planned review. For imprecision, if we obtain pooled estimates of effects with wide CI (a situation which occurs when included studies have small number of participants and experience few events), we will rate down the certainty of the evidence. Finally, we will downgrade the certainty of evidence of effects of fIPV if our funnel plots show a high likelihood of publication bias.

\section{Patient and public involvement}

Patients were not involved in the design of this study.

\section{Ethics and dissemmination}

Collection of data for our review does not involve direct contact with human participants. Instead, we will use published and publicly accessed data. The findings of this review will provide policy-makers, health workers and donors with evidence for decision-making with regards to IPV dosage. In the face of the current shortages, this might improve immediate and long-term access to the vaccine. The results of the review will be published in the University of Cape Town online library and in a peer reviewed journal.

\section{Author affiliations}

${ }^{1}$ Department of Epidemiology and Biostatistics, School of Public Health and Family Medicine, Faculty of Health Sciences, University of Cape Town, Cape Town, Western Cape, South Africa

${ }^{2}$ Cochrane South Africa, South African Medical Research Council, Tygerberg, Western Cape, South Africa

${ }^{3}$ Department of Medicine, University of Cape Town, Faculty of Medicine, Cape Town, Western Cape, South Africa

${ }^{4}$ Health Sciences Library, University of Cape Town, Cape Town, Western Cape, South Africa

${ }^{5}$ Division of Epidemiology and Biostatistics,Department of Global Health, Faculty of Medicine and Health Sciences, Stellenbosch University, Cape Town, Western Cape, South Africa

Contributors TRM led the development of the protocol, wrote the first draft, coordinated and integrated comments from co-authors, approved the final version for publication and is the guarantor of the manuscript. TRM and MS developed the search strategy. DEN, KRD and MS critically revised successive drafts of the manuscript, provided important intellectual input and approved the final version of the manuscript. CSW conceived the study, provided supervision and mentorship to TRM, critically revised successive drafts of the manuscript, provided important intellectual input and approved the final version of the manuscript.

Funding This work is supported by the South African Medical Research Council (salaries for Thandiwe Mashunye, Duduzile Ndwandwe, Kopano Dube and Charles S. Wiysonge) and the National Research Foundation of South Africa (Grant numbers: 106035 and 108571).

Competing interests None declared.

Patient consent for publication Not required.

Ethics approval All procedures were approval by the University of Cape Town Human Research Ethics Committee (HREC REF: 412/2018).

Provenance and peer review Not commissioned; externally peer reviewed.

Data sharing statement No additional data are available. 
Open access This is an open access article distributed in accordance with the Creative Commons Attribution Non Commercial (CC BY-NC 4.0) license, which permits others to distribute, remix, adapt, build upon this work non-commercially, and license their derivative works on different terms, provided the original work is properly cited, appropriate credit is given, any changes made indicated, and the use is non-commercial. See: http://creativecommons.org/licenses/by-nc/4.0/.

\section{REFERENCES}

1. Wiysonge CS. Africa is within reach of being declared a polio free region. The Conversation 2015. https://theconversation.com/ africa-is-within-reach-of-being-declared-a-polio-free-region-44786 (Accessed 18 Mar 2018).

2. Kliegman R, Behrman RE, Nelson WE. Nelson textbook of pediatrics. 20 ed. Phialdelphia, PA: Elsevier, 2016.

3. World Health Organisation. Polio vaccines: WHO position paper March, 2016 in the Weekly epidemiological record: 25 March 2016. 2016;91, 12:145-68 http://www.who.int/wer/2016/wer9112.pdf?ua= 1.

4. Okayasu H, Sutter RW, Jafari HS, et al. Affordable inactivated poliovirus vaccine: strategies and progress. J Infect Dis 2014;210(Suppl 1):S459-64.

5. UNICEF Supply Division. OPV Supply \& Outlook. 2013. https://www. unicef.org/supply/files/Oral_polio_vaccine_update.pdfCurrent

6. Burns CC, Diop OM, Sutter RW, et al. Vaccine-derived polioviruses. $J$ Infect Dis 2014;210:S283-93.

7. Platt LR, Estívariz CF, Sutter RW. Vaccine-associated paralytic poliomyelitis: a review of the epidemiology and estimation of the global burden. J Infect Dis 2014;210(Suppl 1):S380-9.

8. Global Polio Eradication Initiative. Polio Eradication \& Endgame Strategy 2013-2018. 2013 http://polioeradication.org/wpcontent/ uploads/2016/07/PEESP_EN_A4.pdf.

9. Zipursky S on behalf of the IMG Polio Partners' Group. Presentation on the Update on the switch and IPV supply, Geneva. 2016 http://polioeradication.org/wp-content/uploads/2016/07/PPG_ Mtg20160624_Presentation2.pdf (Accessed on 11 Jan 2018).

10. Meeting of the Strategic Advisory Group of Experts on Immunization, November 2012 - conclusions and recommendations in the Weekly epidemiological record. 2013;88:1-16 http://www.who.int/wer/2013/ wer8801.pdf.

11. Anand A, Molodecky NA, Pallansch MA, et al. Immunogenicity to poliovirus type 2 following two doses of fractional intradermal inactivated poliovirus vaccine: A novel dose sparing immunization schedule. Vaccine 2017;35:2993-8.

12. . UNICEF Supply Division: Inactivated Polio Vaccine Supply and Demand Update, 2016. https://www.unicef.org/supply/files/ Inactivated_Polio_Vaccine_(IPV)_-_september_2016.pdf (Accessed 11 Jan 2018)

13. GAVI and GPEI IPV Supply and Procurement Roadmap In support of the Polio Eradication Endgame Strategy Update August 2017: 4 The Market Shaping Goal. http://www.gavi.org/library/gavi./supplyprocurement/ipv-roadmap-public-summary/ (accessed on 11 Jan 2018).

14. UNICEF. Data showing awarded price per dose (in US\$ or EUR) per product per supplier per calendar year, based on supply agreements. https://www.unicef.org/supply/files/IPV.pdf (Accessed on 11 Jan 2018).

15. Hampton LM, Farrell M, Ramirez-Gonzalez A, et al. Cessation of trivalent oral poliovirus vaccine and introduction of inactivated poliovirus vaccine - worldwide, 2016. MMWR Morb Mortal Wkly Rep 2016;65:934-8.

16. Lewis I, Ottosen A, Rubin J, et al. A supply and demand management perspective on the accelerated global introductions of inactivated poliovirus vaccine in a constrained supply market. $J$ Infect Dis 2017;216(suppl_1):S33-9.

17. Global Polio Eradication Initiative. Use of fractional dose IPV in routine immunization programmes: Considerations for decisionmaking. 2017. http://www.who.int/immunization/diseases/ poliomyelitis/endgame_objective2/inactivated_polio_vaccine/fIPV considerations_for_decision-making_April2017.pdf (Accessed 11 Jan 2018).

18. UNICEF. Update on IPV introduction and use of fractional dose given intradermal with $0.1 \mathrm{ml}$ syringe. $2017 \mathrm{https}$ ://www.unicef.org/supply/ files/6a._Fractional_IPV_and_syringes.pdf (Accessed 11 Jan 2018).
19. Global Polio Eradication Initiative. Post-Certification Strategic Plan (Review draft). 2017. http://www.who.int/immunization/sage/ meetings/2017/october/4_polio_Post_Certification_Strategic_Plan_ Draft_Web.pdf (accessed 23 Nov 2017).

20. Resik S, Tejeda A, Lago PM, Mas Lago P, et al. Randomized controlled clinical trial of fractional doses of inactivated poliovirus vaccine administered intradermally by needle-free device in Cuba. $J$ Infect Dis 2010;201:1344-52.

21. Mohammed AJ, AIAwaidy S, Bawikar S, et al. Fractional doses of inactivated poliovirus vaccine in Oman. N Engl $\mathrm{J}$ Med 2010;362:2351-9.

22. Resik S, Tejeda A, Sutter RW, et al. Priming after a fractional dose of inactivated poliovirus vaccine. $N$ Engl J Med 2013;368:416-24.

23. Anand A, Zaman K, Estívariz CF, et al. Early priming with inactivated poliovirus vaccine (IPV) and intradermal fractional dose IPV administered by a microneedle device: A randomized controlled trial. Vaccine 2015;33:6816-22.

24. Lambert PH, Laurent PE. Intradermal vaccine delivery: will new delivery systems transform vaccine administration? Vaccine 2008;26:3197-208.

25. Okayasu $\mathrm{H}$, Sein $\mathrm{C}$, Chang Blanc D, et al. Intradermal Administration of Fractional Doses of Inactivated Poliovirus Vaccine: A DoseSparing Option for Polio Immunization. J Infect Dis 2017;216(suppl_1 ):S161-S167.

26. Bahl S, Verma H, Bhatnagar $\mathrm{P}$, et al. Fractional-dose inactivated poliovirus vaccine immunization campaign - telangana state, India, June 2016. MMWR Morb Mortal Wkly Rep 2016;65:859-63.

27. United Nations. The 2030 agenda for sustainable development. 2015. https://sustainabledevelopment.un.org/content/documents/ $21252030 \% 20$ Agenda\%20for\%20Sustainable\%20Development\% 20web.pdf (Accessed 13 Jan 2018)

28. Grassly NC. Immunogenicity and effectiveness of routine immunization with 1 or 2 doses of inactivated poliovirus vaccine: systematic review and meta-analysis. J Infect Dis 2014;210 Suppl 1:S439-S446.

29. Wiysonge CS, Kamadjeu R, Tsague L. Systematic reviews in context: highlighting systematic reviews relevant to Africa in the Pan African Medical Journal. Pan Afr Med J 2016;24:180.

30. Guyatt GH, Oxman AD, Vist GE, et al. GRADE: an emerging consensus on rating quality of evidence and strength of recommendations. BMJ 2008;336:924-6.

31. Lee G, Carr W. ACIP Evidence-Based Recommendations Work GroupACIP Evidence Based Recommendations Work Group. Updated Framework for Development of Evidence-Based Recommendations by the Advisory Committee on Immunization Practices. MMWR Morb Mortal Wkly Rep 2018;67:1271-2.

32. Higgins JPT, Green S. Cochrane handbook for systematic reviews of interventions Version 5.1.0 [updated March 2011]: The Cochrane Collaboration. 2011. http://handbook.cochrane.org (Accessed 20 Nov 2017).

33. Sterne JA, Hernán MA, Reeves BC, et al. ROBINS-I: a tool for assessing risk of bias in non-randomised studies of interventions. BMJ 2016;355:i4919.

34. Higgins JP, Thompson SG, Deeks JJ, et al. Measuring inconsistency in meta-analyses. BMJ 2003;327:557-60.

35. The World Bank Group. Data. Countries an Economies. https://data. worldbank.org/country/ (Accessed 2 Feb 2018).

36. Sterne JA, Sutton AJ, loannidis JP, et al. Recommendations for examining and interpreting funnel plot asymmetry in meta-analyses of randomised controlled trials. BMJ 2011;343:d4002.

37. Welch V, Petticrew M, Tugwell P, et al. PRISMA-Equity 2012 extension: reporting guidelines for systematic reviews with a focus on health equity. PLoS Med 2012;9:e1001333.

38. Moher D, Shamseer L, Clarke M, et al. Preferred reporting items for systematic review and meta-analysis protocols (PRISMA-P) 2015 statement. Syst Rev 2015;4:1):1.

39. Shamseer L, Moher D, Clarke M, et al. Preferred reporting items for systematic review and meta-analysis protocols (PRISMA-P) 2015: elaboration and explanation. BMJ 2015;349:g7647.

40. Guyatt G, Oxman AD, Akl EA, et al. GRADE guidelines: 1. Introduction-GRADE evidence profiles and summary of findings tables. J Clin Epidemiol 2011;64:383-94.

41. Balshem $\mathrm{H}$, Helfand M, Schünemann $\mathrm{HJ}$, et al. GRADE guidelines: 3 . Rating the quality of evidence. J Clin Epidemiol 2011;64:401-6. 